The Cyclopedia of Practical Surgery; including an Etymological and Critical Terminolony; Description of Instruments; a copious Bibliography, and an Historical Fiew of the Progress of the Science to the present day. The whole presenting a complete digest of the Doctrines and Practice of Surgery. By WILtian B. Costello, assisted by numerous distinguished Writers. 4 vols. imp. 8vo. Profusely illustrated. London: Taylor and Greening. Is61.

THIs large work, commenced many years ago, and for the last ten occupying Dr. Costello's undivided attention, has been completed within the last few months. Indeed, so many years have elapsed since the publication of the earlier parts, that at one time it was believed that all idea of its continuation had been abandoned.

It consists of thirty parts, or 3000 pages in double columns; and, differing from many other dictionaries, it embraces every known subject in relation to surgery, with the advantage of copious illustration by means of 1500 well-executed engravings on wood and steel, this last feature being readily permitted by the size of the work.

Among the contributions to the first volume we find Abdomen and Ablation, by the late Bransby Cooper; Acupuncture, by Dr. Elliottson; Morbid Anatomy, by Dr. Hodgkin; Angeioleucitis, by Velpeau; and Blister, Burns and Scalds, Caustics, Cauterization, and Cicatrix, by Mr. Ure. In this volume, also, there are articles by Dieffenbach, T. Bell, F. Tyrrell, Dr. Wardrop, J. Blackburn, Dr. Little, H. T. Chapman, Middlemore, W. Fergusson, Wells, Wharton Jones, and others. Sir Astley Cooper is the author of Castration, Dr. Walshe of Cancer and Cephalhæmatoma, and Dr. Wardrop of Bloodletting. Many of these articles, although now written some years, have by no means lost their value and importance, and several of the subjects are more fully treated than in some of our latest works.

The succeeding three volumes contain subjects that are pretity well brought up to the standard of the present day. In the second, Hydrocele is from the pen of Sir Benjamin Brodie; Gun-shot Wounds, by Sir John Liddell; Ectopia, Emphysema, Empyema, Encephalocele, and Endo-osteitis, by Dr. Walshe; Head, by John Adams; Intestinal Fistula and Hernia, by T. P. Teale; and Larynx, by the late F. Ryland. Mr. Coulson is the author of Hip-joint; J. Erichsen, of Hæmorrhoids; Jobert de Lamballe, of Intestines; Alph. A mussat, of Irrigation; Ure, of Inflammation; John Gay, of Lips; and Dr. P. Rayer, of Farcy.

In the third volume Maisonneave contributes the article Luxation; John Birkett, that of Mamma; Dr. Charles Campbell, that of Parturition; Dr. John Reid, that of Pregnancy; and Desormeaux, that of Ovary. Osteitis, Osteophymy, Necrosis, and Perchloride of Iron, are by Dr. Paul Broca; Pain is by the late Dr. John Snow; Moxa, by Mr. Ure; Nævus, by Dr. Collin ; Esophagus, by Chelius and the author.

Among the names of the contributors to the fourth volume are-Herbert Shelley, Bouvier and Denos, Dr. Gallard, V. Foncher, and Paul Broca. Throughout all the volumes, but especially the last, many of the articles are by Dr. Costello, who has rendered them complete with the latest discoveries in surgical science, whether in this country or elsewhere. Indeed, we may justly assert that many of the peculiarities of both German and French practice, not commonly found in other books, are carefully detailed in the present work. It would be impossible to enter into an analysis of any of the articles; the names of the authors are vouchers for their worth. We may observe, nevertheless, that the great advances made in the knowledge of Morbid Anatomy and Pathology have not been either omitted or overlooked; and, to use the author's words in his address, "Dr. Broca, of Paris, has contributed an exposition of this great and important subject (Tumours) that embodies some of the most advanced views of pathological knowledge." This we quote as an instance in illustration.

In conclusion, we may remark that there are many subjects treated for the first time in our language in this Cyclopædia; this will be apparent on going through the volumes. They constitute a connected series of monographs, alphabetically arranged, replete with information in every department of Surgery; forming a library for the practitioner and the student. Dr. Costello has accomplished a labour of great magnitude, one that will ever be associated most creditably and honourably with his name. His Cyclopædia of Practical Surgery will rank with that of Anatomy and Physiology by Dr. Todd, and with Copland's Dictionary of Medicine, both monuments of labour and research. We can recommend Dr. Costello's work as a comprehensive dictionary of all branches of Surgery, embracing almost everything relating to the subject.

\section{The Technologist.}

THE number for April of this useful periodical comes before us much enlarged. Amongst the contributions is one of great interest upon the Manufacture of Kelp, which has considerably revived of late years in consequence of the large demand for iodine for photographic purposes. Another paper is a record of the most remarkable specimens of native gold, comprising as many as 150 , the largest weighing 2217 ounces, and which was sold at Ballarat in 1858 for $£ 10,500$. It appears also that the purest nuggets, like native silver and iron, are never found absolutely free from alloy.

\section{QUARANTINE REGULATIONS: CONTAGIOUS DISEASES.}

To the Editor of THe LAvicer.

SIR, - A few weeks ago, through the medium of your widelycirculated journal, I directed attention to our quarantine regulations, and asked for some explanation of what must have struck any reasonable person as a great anomaly, referring to the facts that small-pox, a disease contagious in all climes, is permitted importation without check, whilst ships arriving with cases of yellow fever on board have been subjected to quarantine. About the contagiousness of the former there is no difference of opinion. As regards the latter, the highest authorities, numbering amongst them men who have had extensive opportunities of observing the disease, entirely repudiate the doctrine that it is disseminated by contagion, but trace its origin and progress to the influence of dirty cities, dirty, illventilated, ill-provisioned ships, aided by a high temperature and a peculiar condition of the atmosphere. What has been ohserved relative to the causes of the origin and progress of yellow fever applies with equal force to cholera, plague, gastric and other low forms of fever, which latter we are constantly witnessing in our cities and towns, and now so aptly denominated "dirt fever."

The opinion that the atmospheric influence is inoperative in producing either disease in a bad, if in any, form without the co-operating powers produced by dirt, and in a greater or less degree its invariable concomitants, is now regarded by most practical men as beyond question.

However, if we simply take a common-sense view of the monstrous inconsistency in connexion with the present quarantine system, no one doubts that small-pox spreads by contagion; whilst it can be as boldly asserted that yellow fever has been frequently imported into this country, and that it has never been known to disseminate itself here, either by contagion or in any other way. Hence the inutility-if not something worse- of encouraging prejudices calculated to produce many evils; and perhaps amongst the worst, that of affording to foreign governments a powerful excuse for enforcing an improper system of quarantine.

The spread of small-pox in the Southampton Poor-house, attributed to the importation of the disease from the Tuscarora, (as seen by a paragraph in the local papers, one of which I have forwarded to you, ) is the reason for my again introducing the subject to your attention, hoping thereby to invoke your powerful aid in the reformation of a defective system.

I am, Sir, yours obediently,

Sonthampton, April, 1862. EDwiN HeARNe, M.B. Lond. 